\title{
PERFORMANCE EVALUATION USING MANDELBROT IMAGES FOR IMAGE REGISTRATION ALGORITHMS
}

\author{
Koichi Ito ${ }^{1}$, Ayako Suzuki ${ }^{1}$, Sei Nagashima ${ }^{2}$ and Takafumi Aoki ${ }^{1}$ \\ ${ }^{1}$ Graduate School of Information Sciences, Tohoku University, \\ Sendai-shi 980-8579, Japan. \\ E-mail: ito@aoki.ecei.tohoku.ac.jp \\ ${ }^{2}$ Yamatake Corporation, Fujisawa-shi, 251-8522, Japan.
}

\begin{abstract}
High-accuracy image registration is an important fundamental task in many fields, such as image sensing, image/video processing, computer vision, etc. In order to evaluate accuracy of image registration algorithms, the reference images transformed with known parameters have to be used. Reference images taken by a camera may include human errors, while reference images generated by a computer may require pixel interpolation in the process. To address these problems, this paper proposes a performance evaluation method using the Mandelbrot set which is one of the famous fractals. Experimental evaluation shows effectiveness of the proposed method.
\end{abstract}

Index Terms - image registration, image matching, performance evaluation, Mandelbrot set, phase-only correlation

\section{INTRODUCTION}

High-accuracy image registration is an important fundamental task in many fields, such as image sensing, image/video processing, computer vision, etc [1]. In particular, image registration techniques with sub-pixel accuracy have been receiving much attention. Image registration algorithms are broadly classified into 2 categories: feature-based and area-based algorithms. The feature-based algorithms employ feature points on images to align images, e.g., SIFT (Scale Invariant Feature Transform) [2]. On the other hand, the areabased algorithms employ similarity (or dissimilarity) measures between images, e.g., SAD (Sum of Absolute Differences), SSD (Sum of Squared Differences) [3, 4], and Phase-Only Correlation (POC) $[5,6,7]$.

In order to evaluate accuracy of image registration algorithms, the reference images transformed with known parameters have to be used. For example, to obtain reference images with sub-pixel translations, we apply a lowpass filter to a high-resolution image and downsample shifted versions of the image. Using appropriate downsampling rates, we can generate images having sub-pixel translations. However, pixel interpolation is required to generate images with rotation and scale. We can also obtain translated, rotated and scaled images, where an object is mounted on a micro stage which allows precise alignment of the object position and is taken by a camera. In this case, we need a precise micro stage and carefully operate the camera and stage to reduce the error associated with human and device.

In this paper, we propose a performance evaluation method using the Mandelbrot set [8] for image registration algorithms. Figure 1 shows an example of the Mandelbrot set. The Mandelbrot set is a set of points in the complex plan having elaborate boundaries, where

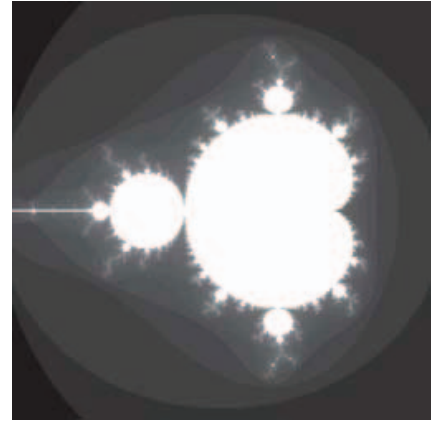

Fig. 1. Mandelbrot set.

the little copies of the Mandelbrot set are connected to the main body of the set and are all slightly different. Considering the Mandelbrot set as two-dimensional (2D) signals defined in continuous space, we can generate images transformed with arbitrary parameters without interpolating pixels, since the Mandelbrot set has infinite resolution. We also demonstrate effectiveness of the proposed method through experiments for evaluating performance of registration algorithms.

\section{MANDELBROT IMAGE GENERATION}

This section describes a Mandelbrot image generation procedure. The Mandelbrot set defined as the set of complex values of $x_{1}+j x_{2}$ is obtained from the following quadratic recurrence equation,

$$
z_{n+1}=z_{n}^{2}+x_{1}+j x_{2},
$$

where $x_{1}$ and $x_{2}$ are real-number coordinates, and the initial condition is $z_{0}=0$. In nature, the Mandelbrot set is the set of all points in the complex plain $x_{1}+j x_{2}$ which does not diverge under $n \rightarrow \infty$. In order to reduce the computational cost, we calculate Eq. (1) up to $n=U$, where $U=1,000$ in this paper. If $\left|z_{n}\right| \geq 2(n \leq U)$, the intensity value $h_{c}\left(x_{1}, x_{2}\right)$ for $\left(x_{1}, x_{2}\right)$ in the complex plain is $n$. If $\left|z_{n}\right| \leq 2$ until $n=U$, the intensity value $h_{c}\left(x_{1}, x_{2}\right)$ is $U$. Thus, we can obtain the Mandelbrot set as $h_{c}\left(x_{1}, x_{2}\right)$. Note that we employ $h_{c}^{\prime}\left(x_{1}, x_{2}\right)=\log \left\{h_{c}\left(x_{1}, x_{2}\right)+1\right\}$ instead of $h_{c}\left(x_{1}, x_{2}\right)$ for intensity compression.

The Mandelbrot set $h_{c}^{\prime}\left(x_{1}, x_{2}\right)$ can be considered as a 2D image defined in continuous space. We now sample a continuous Mandelbrot set $h_{c}^{\prime}\left(x_{1}, x_{2}\right)$ at the sampling intervals $T_{1}$ and $T_{2}$ to have 


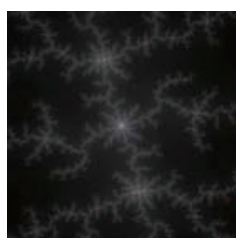

A $(l=0)$

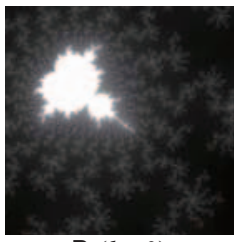

B $(l=0)$

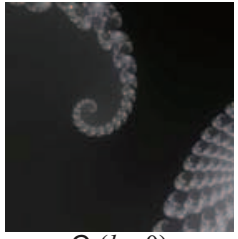

C $(l=0)$

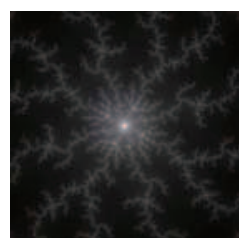

A $(l=1)$

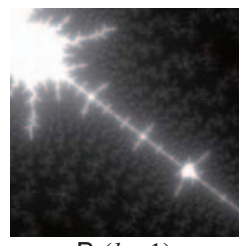

B $(l=1)$

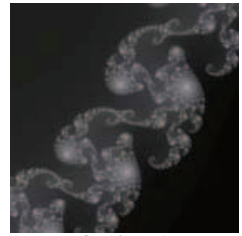

C $(l=1)$

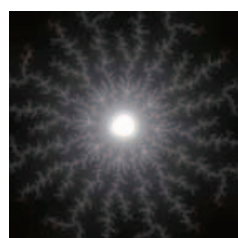

A $(l=2)$

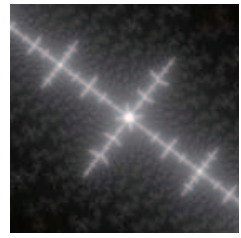

B $(l=2)$

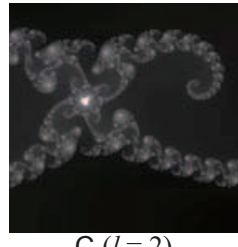

C $(l=2)$
Fig. 2. Examples of Mandelbrot images

a Mandelbrot image $h\left(n_{1}, n_{2}\right)$. Let $\left(c_{1}, c_{2}\right)$ be image centers and $\left(n_{1}, n_{2}\right)$ be discrete space indices, we have

$$
h\left(n_{1}, n_{2}\right)=\left.h_{c}^{\prime}\left(x_{1}-c_{1}, x_{2}-c_{2}\right)\right|_{x_{1}=n_{1} T_{1}, x_{2}=n_{2} T_{2}},
$$

where $n_{1}=-M_{1}, \cdots, M_{1}$ and $n_{2}=-M_{2}, \cdots, M_{2}$. Figure 2 shows examples of Mandelbrot images generated by changing image centers $\left(c_{1}, c_{2}\right)$ and a scale factor. In these figures, we employ

$$
\begin{array}{ll}
\mathrm{A}: & D=1.0 \times 10^{-11}, \\
& c_{1}=-0.25272149866535, \\
& c_{2}=0.84996890117939, \\
\mathrm{~B}: \quad & D=1.0 \times 10^{-7}, \\
c_{1} & =-0.64868627955000, \\
& c_{2}=0.48617790435000, \\
\mathrm{C}: \quad & D=5.0 \times 10^{-6}, \\
c_{1} & =0.28950114650000, \\
c_{2} & =0.01346307350000,
\end{array}
$$

where $T_{1}=T_{2}=D \times 10^{l}(l=0,1,2)$ and $M_{1}=M_{2}=200$. We can obtain various versions of Mandelbrot images by changing the viewpoint of the Mandelbrot set as shown in Fig. 2.

In the following, we describe how to obtain the Mandelbrot images transformed with arbitrary parameters. Consider $f_{c}\left(x_{1}, x_{2}\right)$ as a 2D Mandelbrot set in continuous space with real-number indices $x_{1}$ and $x_{2}$. Let $g_{c}\left(x_{1}, x_{2}\right)$ be the Mandelbrot set obtained by translating, rotating and scaling $f_{c}\left(x_{1}, x_{2}\right)$ by the translations $\left(\delta_{1}, \delta_{2}\right)$, the angle $\theta$ and the scaling factor $s$, respectively. Assume that $f\left(n_{1}, n_{2}\right)$ and $g\left(n_{1}, n_{2}\right)$ are spatially sampled images of $f_{c}\left(x_{1}, x_{2}\right)$ and $g_{c}\left(x_{1}, x_{2}\right)$ as

$$
\begin{aligned}
f\left(n_{1}, n_{2}\right)= & \left.f_{c}\left(x_{1}, x_{2}\right)\right|_{x_{1}=n_{1} T_{1}, x_{2}=n_{2} T_{2}} \\
g\left(n_{1}, n_{2}\right)= & \left.g_{c}\left(x_{1}, x_{2}\right)\right|_{x_{1}=n_{1} T_{1}, x_{2}=n_{2} T_{2}} \\
= & f_{c}\left(s\left(x_{1}-\delta_{1}\right) \cos \theta-s\left(x_{2}-\delta_{2}\right) \sin \theta\right. \\
& s\left(x_{1}-\delta_{1}\right) \sin \theta+s\left(x_{2}-\delta_{2}\right) \\
& \times \cos \theta)\left.\right|_{x_{1}=n_{1} T_{1}, x_{2}=n_{2} T_{2}} .
\end{aligned}
$$
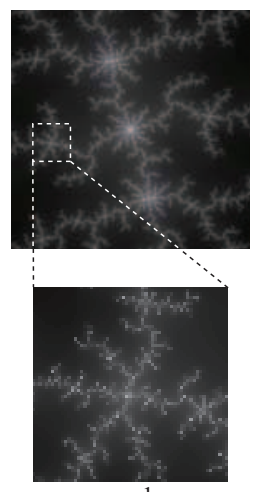

$m=1$
(a)
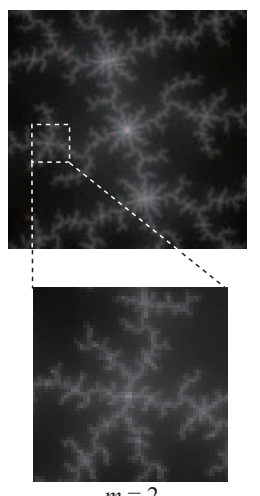

(b)
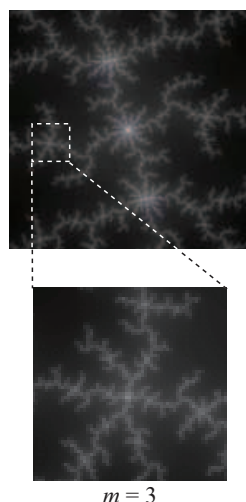

(c)
Fig. 3. Examples of Mandelbrot images reducing the effect of aliasing: (a) $m=1$ (original), (b) $m=2$ and (c) $m=3$.

Note that we consider here the similarity transformation for simplicity. We can also employ nonlinear transformation to generate the Mandelbrot images. The generated Mandelbrot images contain aliasing, which prevents accurate performance evaluation of image registration algorithms. To reduce the effect of aliasing, we apply a lowpass filter to a high-resolution image having $m$-times higher resolution. Figure 3 shows examples of Mandelbrot images with and without anti-aliasing.

\section{EXPERIMENTS AND DISCUSSION}

This section presents experiments for evaluating performance of image registration algorithms using Mandelbrot images.

\subsection{Image Registration Algorithms}

In the experiments, we employ two image registration algorithms. Algorithm (A) is an image registration algorithm using Phase-Only Correlation (POC) proposed by [6], which can estimate translation, rotation and scale. Algorithm (B) is the extended version of Algorithm (A) which estimates transformation parameters iteratively. In general, there is a non-overlapped area between two images with image transformation. The non-overlapped area becomes the uncorrelated noise components in the POC function and then reduce the estimation accuracy of Algorithm (A). Addressing this problem, we detect the common areas between two images and estimate transformation parameters between detected common areas using POC. Repeating the above procedure, we can improve estimation accuracy even if images are translated, rotated and scaled. In this paper, the number of iteration is 3 .

\subsection{Experiments and Discussion}

We carry out two experiments in this paper; one is to evaluate the effect of aliasing and the other is to evaluate performance of image registration algorithms using translated, rotated and scaled images.

In the first experiment, we generate the Mandelbrot images, where $m$ is changed from 1 to 3 . So, the maximum image resolution considered in this experiment is 3-times higher than that of original Mandelbrot images. In order to compare the images used in [6], we generate the Mandelbrot images under the same condition summarized as follows, 


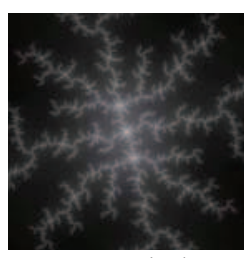

$\delta_{1}=0$ pixel

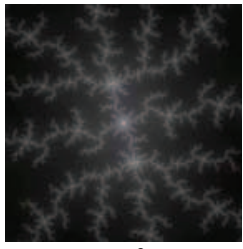

$\theta=0^{\circ}$

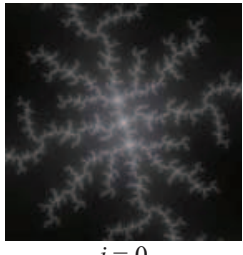

$i=0$

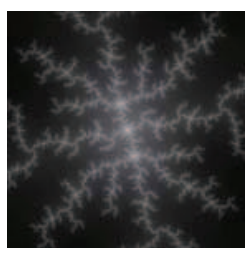

$\delta_{1}=2.5$ pixel

(a)

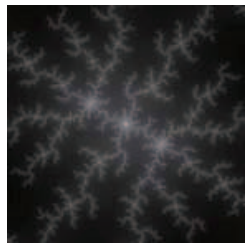

$\theta=45^{\circ}$

(b)

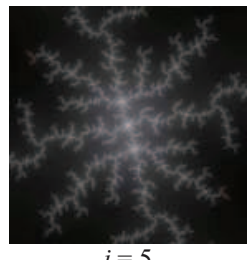

(c)

Fig. 4. Examples of Mandelbrot images $(m=3)$ : (a) translation, (b) rotation and (c) scale.

- Translation: Images translated horizontally from 0 to 5 pixels with a spacing 0.1 pixels, where the total number of images is 51 .

- Rotation: Images rotated from $0^{\circ}$ to $90^{\circ}$ with a rotation spacing $1^{\circ}$, where the total number of images is 91 .

- Scale: Images scaled to $500 /(500+5 i)(i=0, \cdots, 11)$, where the total number of images is 12 . In [6], the wood cube mounted on the $z$-stage is taken by a camera while moving the stage 12 times with each micro step $5 \mathrm{~mm}$, where the initial distance from the camera to the cube is about $50 \mathrm{~cm}$.

We employ the parameters for Mandelbrot image generation as follows: $T_{1}=T_{2}=10^{-11}, c_{1}=-0.25272149866535, c_{2}=$ 0.84996890117939 and the image size is $401 \times 401$ pixels $(M=$ $200)$. Figure 4 shows examples of Mandelbrot images $(m=3)$. The estimation accuracy is evaluated by errors between the estimate and true values. The RMS (Root Mean Square) error calculated from all the errors is also employed to evaluate performance of the registration algorithms.

Figure 5 shows the estimation errors for translation, rotation and scale, respectively, and Table 1 shows the RMS errors. As a result, the errors for the Mandelbrot images with $m=1$ are larger than those of other images, since the Mandelbrot images with $m=1$ contain aliasing. The errors for the Mandelbrot images with $m=3$ are comparable with that of the wood cube. As is observed in this experiment, we need to generate the anti-aliased Mandelbrot images from the Mandelbrot images having 3-times higher resolution for accurate performance evaluation of image registration algorithms.

In the second experiment, we generate 3 types of the Mandelbrot images, where we employ the parameter set used in Fig. 2.

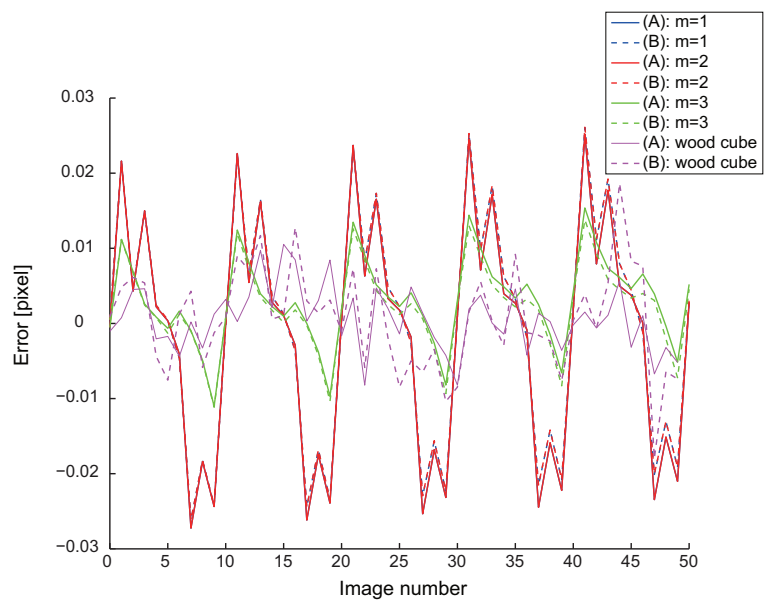

(a)

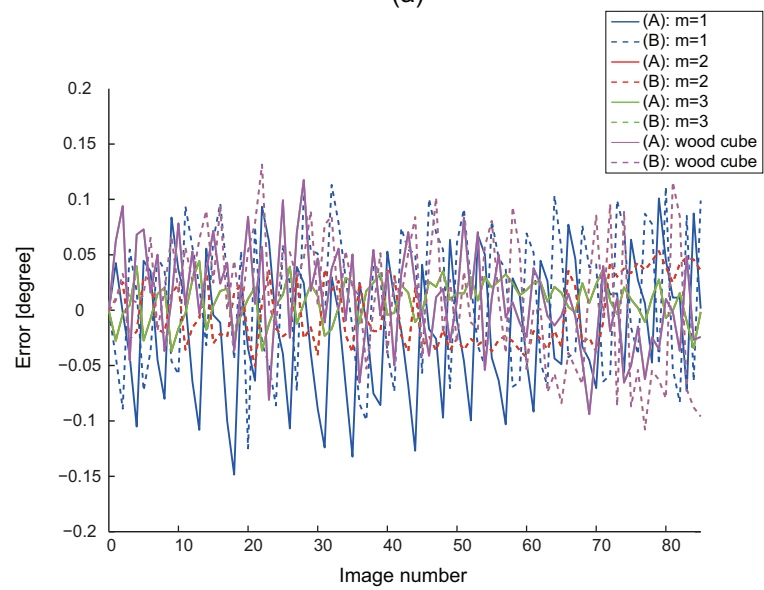

(b)

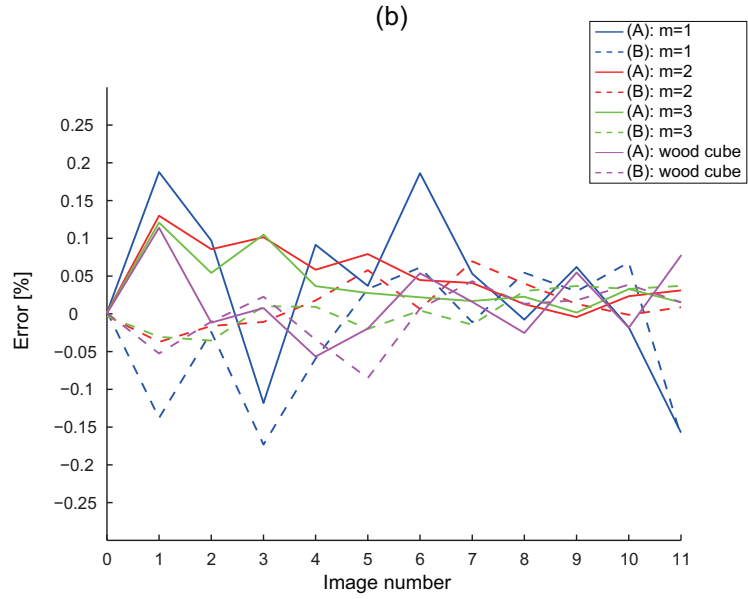

(c)

Fig. 5. Estimation errors: (a) translation, (b) rotation and (c) scale.

Each Mandelbrot image is transformed according to the parameter set described in the upper rows of Table 2 . Note that $\left(\delta_{1}, \delta_{2}\right)$ indicate translational displacements, $\theta$ indicates a rotation angle and $s$ indicates a scaling factor. Figure 6 shows the Mandelbrot images 


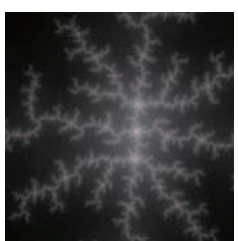

A1

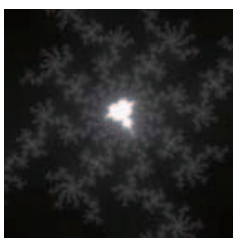

B1

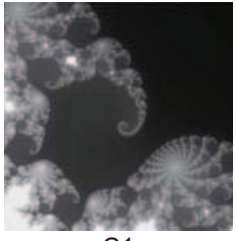

C1

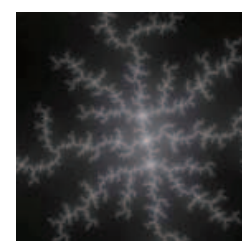

A2

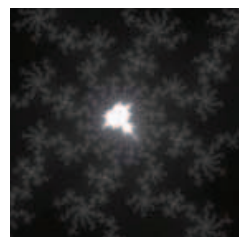

B2

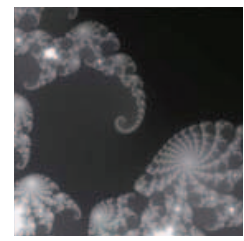

C2
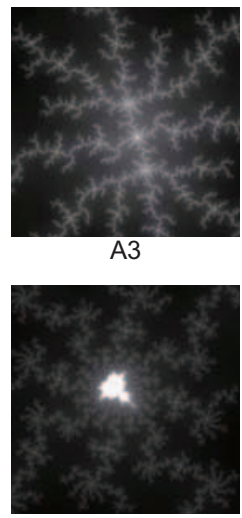

B3

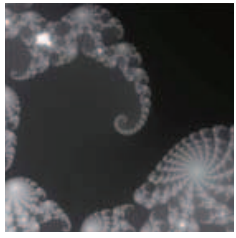

C3
Fig. 6. Translated, rotated and scaled Mandelbrot images

Table 1. RMS errors

\begin{tabular}{c|ccccc}
\hline & & $m=1$ & $m=2$ & $m=3$ & Wood cube \\
\hline Translation & (A) & 0.0196 & 0.0152 & 0.0065 & 0.0042 \\
\cline { 2 - 6 } [pixel] & (B) & 0.0191 & 0.0148 & 0.0061 & 0.0068 \\
\hline Rotation & (A) & 0.0618 & 0.0204 & 0.0204 & 0.0455 \\
\cline { 2 - 6 }$[$ degree] & (B) & 0.0650 & 0.0299 & 0.0299 & 0.0586 \\
\hline Scale & (A) & 0.1060 & 0.0643 & 0.0526 & 0.0547 \\
\cline { 2 - 6 }$[\%]$ & $(\mathrm{B})$ & 0.0880 & 0.0319 & 0.0254 & 0.0375 \\
\hline
\end{tabular}

used in this experiment. The image registration algorithm used in this experiment is Algorithm (B). The middle and lower rows of Table 2 show the estimate values obtained from Algorithm (B) and the errors between true and estimate values, respectively. As a result, the estimate values include only a small error. Thus, we can accurately generate the Mandelbrot images transformed with the arbitrary parameters from the Mandelbrot set. The Mandelbrot images are useful for evaluating performance of the image registration algorithms.

\section{CONCLUSION}

This paper has proposed a performance evaluation method using the Mandelbrot set which is one of the famous fractals. Experimental evaluation using the Mandelbrot images shows effectiveness of the proposed method. In future, we will consider a universal performance evaluation method using the Mandelbrot images or other fractal images for image registration algorithms.

\section{REFERENCES}

[1] B. Zitová and J. Flusser, "Image registration methods: A survey," Image and Vision Computing, vol. 21, no. 4, pp. 977-1000, 2003.
Table 2. Experimental results (upper rows: true values, middle rows: estimate values and lower rows: errors).

\begin{tabular}{c|ccc}
\hline & $\left(\delta_{1}, \delta_{2}\right)$ & $\theta$ & $s$ \\
\hline & $(28.5039,6.9342)$ & 18.2053 & 1.2000 \\
A1 & $(28.6176,6.7650)$ & 18.1994 & 1.2000 \\
& $(0.1137,-0.1692)$ & -0.0059 & 0.0000 \\
\hline & $(27.6544,22.1462)$ & 5.2880 & 1.1000 \\
A2 & $(27.7056,22.1244)$ & 5.2646 & 1.1004 \\
& $(0.0512,-0.0218)$ & -0.0234 & 0.0004 \\
\hline & $(20.1641,25.1436)$ & 0.5892 & 1.5000 \\
A3 & $(20.0537,25.0300)$ & 0.5517 & 1.5001 \\
& $(-0.1104,-0.1136)$ & -0.0375 & 0.0001 \\
\hline & $(20.4383,11.3844)$ & 24.9539 & 1.1000 \\
B1 & $(20.6272,11.2386)$ & 24.9319 & 1.0996 \\
& $(0.1889,-0.1458)$ & -0.0220 & -0.0004 \\
\hline & $(15.0844,21.2841)$ & 12.8668 & 1.2000 \\
B2 & $(15.1612,21.1849)$ & 12.8455 & 1.2006 \\
& $(0.0768,-0.0992)$ & -0.0213 & 0.0006 \\
\hline & $(9.1385,5.6896)$ & 5.8029 & 1.2000 \\
B3 & $(9.1327,5.5968)$ & 5.8267 & 1.2000 \\
& $(-0.0058,-0.0928)$ & 0.0238 & 0.0000 \\
\hline & $(25.8003,25.6097)$ & 17.8069 & 1.1000 \\
C1 & $(25.9259,25.5092)$ & 17.7722 & 1.1002 \\
& $(0.1256,-0.1005)$ & -0.0347 & 0.0002 \\
\hline & $(10.2591,8.6918)$ & 10.2358 & 1.3000 \\
C2 & $(10.2686,8.5079)$ & 10.2641 & 1.2995 \\
& $(0.0095,-0.1839)$ & 0.0283 & -0.0005 \\
\hline & $(29.6500,17.4838)$ & 12.7049 & 1.5000 \\
C3 & $(29.6454,17.2406)$ & 12.6327 & 1.5001 \\
& $(-0.0046,-0.2432)$ & -0.0722 & 0.0001 \\
\hline & & &
\end{tabular}

[2] D. G. Lowe, "Distinctive image features from scale-invariant keypoints," International Journal of Computer Vision, vol. 60, no. 2, pp. 91-110, 2004.

[3] M. Shimizu and M. Okutomi, "Sub-pixel estimation error cancellation on area-based matching," International Journal of Computer Vision, vol. 63, no. 3, pp. 207-224, 2005.

[4] M. Shimizu and M. Okutomi, "Multi-parameter simultaneous estimation on area-based matching," International Journal of Computer Vision, vol. 67, no. 3, pp. 327-342, 2006.

[5] H. Foroosh, J. B. Zerubia, and M. Berthod, "Extension of phase correlation to subpixel registration," IEEE Trans. Image Processing, vol. 11, no. 3, pp. 188-200, Mar. 2002.

[6] K. Takita, T. Aoki, Y. Sasaki, T. Higuchi, and K. Kobayashi, "High-accuracy subpixel image registration based on phaseonly correlation," IEICE Trans. Fundamentals, vol. E86-A, no. 8, pp. 1925-1934, Aug. 2003.

[7] S. Nagashima, T. Aoki, T. Higuchi, and K. Kobayashi, "A subpixel image matching technique using phase-only correlation," Proc. IEEE 2006 Int. Symp. Intelligent Signal Processing and Communication Systems, pp. 701-704, Dec. 2006.

[8] R. L. Devaney, L. Keen, and K. T. Alligood, Chaos and Fractals: The Mathematics Behind the Computer Graphics, AMS Bookstore, 1989. 